\title{
Poly-lactic-glycolic Acid Microspheres: A Biodegradable Marker for the Diagnosis of Aspiration in Hamsters
}

\author{
CHAIM SPRINGER, SIMON BENITA, YOAV SHERMAN, NESLIHAN GURSOY, DALIA GILHAR, \\ AND AVRAHAM AVITAL \\ Institute of Pulmonology [C.S., A.A.], Department of Pathology [Y.S.], Hadassah-Hebrew University \\ Medical Center, Jerusalem, 91120, Israel; School of Pharmacy [S.B., N.G., D.G.], The Hebrew University \\ of Jerusalem, Jerusalem, 91120, Israel
}

\begin{abstract}
ABSTR
Aspiration is a major cause of lung disease in infants and
young children. As the symptoms and signs of aspiration are not
specific, the diagnosis is delayed due to a low index of suspicion
and low sensitivity and specificity of the available diagnostic
tests. In the present study, we evaluated the utility of micro-
spheres composed of a degradable polymer, polylactic glycolic
acid (PLGA), as a marker to diagnose aspiration in hamsters.
Thirty hamsters underwent direct tracheal instillation of 0.1 mL
of a suspension of PLGA. Eighteen other animals served as
controls and underwent tracheal instillation of 0.1 mL of saline.
Three animals served as naive controls and had no tracheal
instillation. Five animals from the PLGA group and three from
the saline group underwent whole-lung lavage (WLL) on days 1 ,
8, 15, 29, 43, and 58. PLGA microspheres were easily identified
under light microscopy inside the alveolar macrophages obtained
from WLL in all PLGA-instilled animals during all studied days.
\end{abstract}
\section{ABSTRACT}

The number and size of PLGA microspheres within the alveolar macrophages decreased gradually with time with a $90 \%$ rate of disappearance of about $36 \mathrm{~d}$. There was a marked neutrophilic response in lung lavage and a mild peribronchial neutrophil infiltration on the first day after tracheal instillation of PLGA which subsequently disappeared. We conclude that PLGA microspheres are a sensitive and specific marker for aspiration in hamsters. The usefulness of this test in diagnosing aspiration in humans should be further evaluated in clinical studies. (Pediatr Res 58: 537-541, 2005)
Abbreviations
LLAM, lipid-laden alveolar macrophages
PLGA, polylactic glycolic acid
WLL, whole-lung lavage

Aspiration of food or gastric content into the airways is a major cause of lung disease in infants and young children. As the symptoms and signs of aspiration are not specific, the diagnosis is often delayed due to a low index of suspicion of the treating physician and to the low sensitivity and specificity of the available diagnostic tests. Aspiration during swallowing or due to gastroesophageal reflux is common in children suffering from neurologic abnormalities such as cerebral palsy, vocal cord paralysis, neuromuscular diseases, and familial dysautonomia (1-3). Other conditions that can predispose to aspiration in infants and young children include prematurity due to developmental delay of the coordination between swallowing and breathing mechanisms (4), abnormal communication between the airways and the esophagus such as laryngotracheal cleft and tracheoesophageal fistula, and massive gastroesophageal reflux. The definite diagnosis of aspi-

Received October 29, 2004; accepted February 3, 2005

Correspondence to: Chaim Springer, M.D., Institute of Pulmonology, Hadassah University Hospital, POB 12000, Jerusalem 91120, Israel: e-mail: Chaim@ hadassah.org.il

DOI: 10.1203/01.pdr.0000176910.62067.a0 ration is not simple as the available tests such as barium swallow during videofluoroscopy and gastroesophageal scintigraphy are not sensitive enough for the diagnosis of aspiration (5). Also, the oil red $\mathrm{O}$ staining of alveolar macrophages, as suggested by Corwin and Irwin (6), has been shown to be nonspecific for the diagnosis of aspiration (7-9).

Recently, we have shown that polystyrene microspheres instilled directly into the trachea of hamsters can easily be detected within alveolar macrophages obtained from whole lung lavage up to 3 mo after a single tracheal instillation (10). However, as polystyrene is a nondegradable polymer, concerns have been raised as to the safety of this material remaining in the lungs for a long period of time. In the present study, we describe our results using a new degradable marker composed of PLGA microspheres for the diagnosis of aspiration in hamsters.

\section{METHODS}

Animals. Fifty-one female Syrian hamsters (Mesocricetus auratus), aged 9-12 wk, were used. The local Institutional Animal Care and Use Committee approved the study. Thirty hamsters underwent direct tracheal instillation of 
$0.1 \mathrm{~mL}$ of a suspension of PLGA microspheres in phosphate buffer (final concentration $10 \mathrm{mg} / \mathrm{mL}, \mathrm{pH}$ 7.4). Eighteen animals underwent tracheal instillation of $0.1 \mathrm{~mL}$ of normal saline. Three hamsters were used as naive controls (without tracheal instillation). Five animals from the PLGA and three of the saline groups were killed and underwent WLL on days $1,8,15,29,43$, and 58 after tracheal instillation. The naive animals were killed on day 1, and WLL was performed.

PLGA microspheres. PLGA (0.5 g, MW12,000, Birmingham Polymers Sciences Inc., Birmingham, AL) was dissolved in $42 \mathrm{~g}$ acetone. One hundred milliliters $\mathrm{H}_{2} \mathrm{O}$ was added dropwise. Acetone was removed completely under vacuum using a Buchi rotor evaporator $\left(40^{\circ} \mathrm{C}\right)$. An anticaking agent (Poloxamer-188, BASF, Leverkursen, Germany) was added at a concentration of $1.5 \% \mathrm{wt} / \mathrm{wt} \cdot \mathrm{PLGA}$ and Poloxamer 188 are FDA-approved for human use $(11,12)$. The $\mathrm{pH}$ of the dispersion was adjusted with phosphate buffer solution to 7.4. The particle size of the microspheres was determined at room temperature using a noninvasive backscattering high-performance particle size (ALV-NIBS HPPS; Langen, Germany) and an optical imaging microscope. The size of the PLGA microspheres ranged from 1 to $2.4 \mu \mathrm{m}$. The stability of a PLGA microsphere suspension was examined over time in a solution $\mathrm{HCl} 0.2$ $\mathrm{N}(\mathrm{pH} 1.0)$ at $37^{\circ} \mathrm{C}$. The number and size of the PLGA microspheres remained unchanged for at least $21 \mathrm{~d}$.

Anesthesia. A mixture of ketamine $\mathrm{HCl}(50 \mathrm{mg} / \mathrm{mL}, 10 \mathrm{~mL})$ and dehydrobenzperidol $(2.5 \mathrm{mg} / \mathrm{mL}, 2 \mathrm{~mL})$ was administered by intraperitoneal injection, $0.25 \mathrm{~mL}$ before intubation and tracheal instillation and $0.4 \mathrm{~mL}$ before exsanguination. Intubation was performed with a 22 -gauge blunted metal needle. A few breaths were delivered with a small Ambu bag to ascertain intratracheal position of the needle. The PLGA mixture was slowly instilled into the trachea, followed by a few ventilations with an Ambu bag to disperse the fluid and prevent apnea.

Whole-lung lavage. On the day WLL was to be performed, exsanguination was performed by transection of the abdominal aorta. The trachea was cannulated with a 22 gauge blunted needle. Three aliquots of $5 \mathrm{~mL}$ sterile $0.9 \%$ saline were injected and withdrawn with a total recovery of $88 \pm 5 \%$. Five $\mathrm{ml}$ of WLL fluid were centrifuged at 1000 RPM for 10 min and the sediment was suspended in $1 \mathrm{~mL}$ saline. The concentrated WLL fluid was evaluated for total cell counts. Slides were prepared using the Shandon Cytospin 3 (Cheshire, England) and stained by Diff-Quik solutions (Baxter Diagnostics AG, Dudingen, Germany) for differential cell counts and identification of PLGA microspheres in alveolar macrophages.

Microsphere index. One hundred consecutive alveolar macrophages were examined under light microscopy $(\times 400)$. The PLGA microsphere index was expressed as the number of microspheres/100 macrophages.

Histology. After lung lavage was completed, lungs, heart, perihilar lymph glands, liver, and spleen were excised and preserved in formaldehyde. Slides from each organ were prepared for histologic examination. The pathologist (Y.S.) who reviewed the slides of the lungs and other organs was blinded as to the treatment or the control groups.

Statistics. Group data are expressed as mean \pm standard error. Differences between groups were compared using analysis of variance with Bonferroni correction with $p<0.05$ considered significant.

\section{RESULTS}

All animals survived and looked healthy after tracheal instillation of saline or PLGA suspension. After tracheal instillation of saline, there were no statistically significant differences in total cell and differential counts in WLL compared with the naive controls (time 0 in Figure 1 represents data from naive animals). The results of total cell counts as well as neutrophil, macrophage, and lymphocyte counts in WLL fluid after saline and PLGA tracheal instillation are presented in
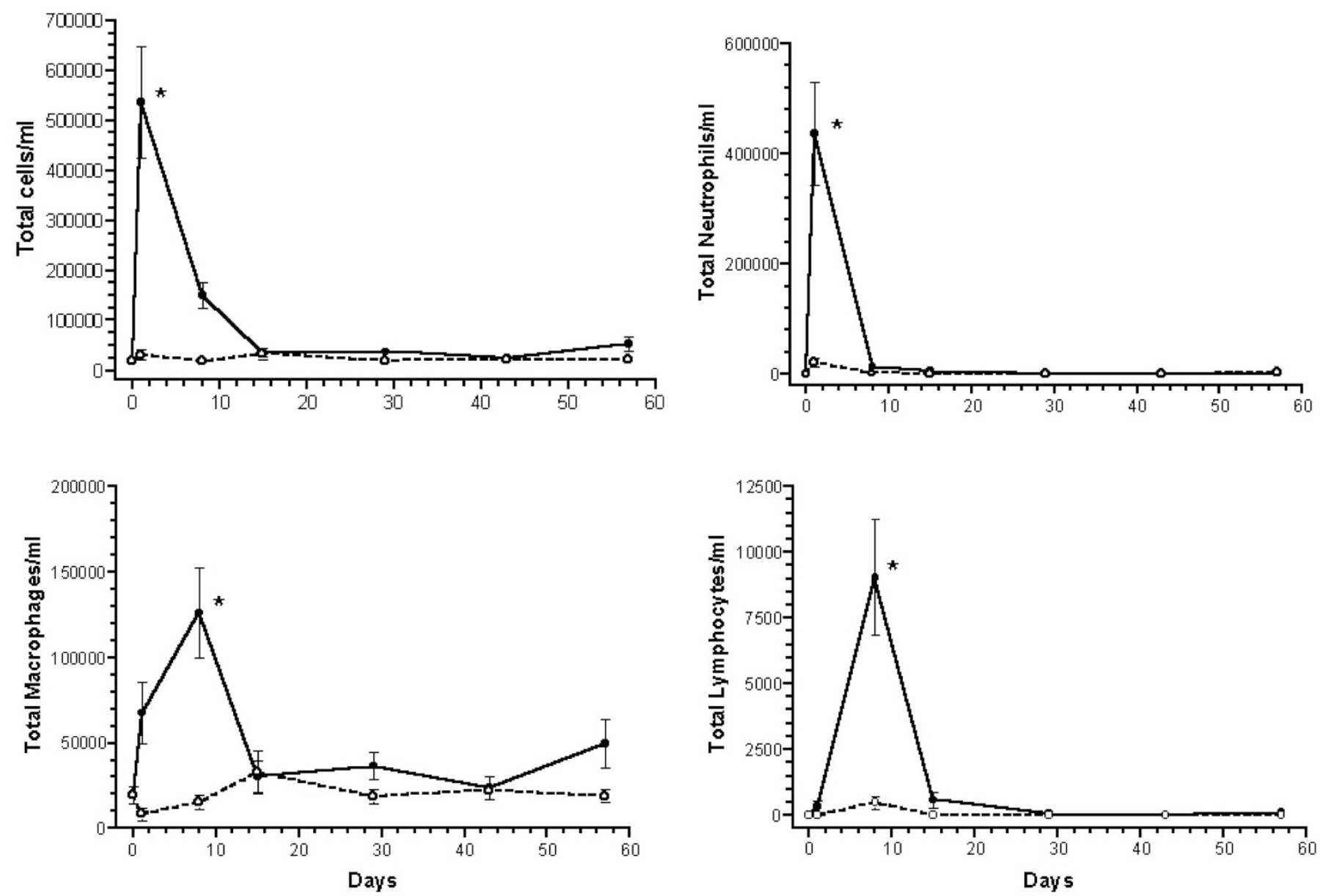

Figure 1. Cell counts in WLL. Mean \pm standard error of the mean of total and differential cell counts in WLL after a single tracheal instillation of saline (dashed line) or PLGA (solid line) suspension in hamsters. *Statistically significant difference $(p<0.001)$ between data obtained from PLGA- and saline-instilled animals. 
Figure 1. After tracheal instillation of PLGA, there was a transient and significant increase in total and differential cell counts compared with the naive and saline-treated animals. This response was maximal on the first day for the total cells and neutrophils: total cell count (mean \pm standard error of the mean): saline-treated animals, $31,000 \pm 10,214$ cells $/ \mathrm{mL}$; PLGA-treated animals, 536,000 $\pm 111,651$ cells/mL $(p<$ 0.001 ) and total neutrophil count: saline-treated animals, $21,526 \pm 7131$ cells/mL; PLGA-treated animals, 436,720 \pm 93,483 cells $/ \mathrm{mL}(p<0.001)$. The maximal increase in macrophage and lymphocyte counts was on the eighth day after tracheal instillation of PLGA: total macrophage count: salinetreated animals, 15,220 \pm 4381 cells $/ \mathrm{mL}$; PLGA-treated animals, $126,320 \pm 26,205$ cells $/ \mathrm{mL}(p<0.001)$ and total lymphocyte count: saline-treated animals, $480 \pm 250$ cells $/ \mathrm{mL}$; PLGA-treated animals: $9040 \pm 2210$ cells $/ \mathrm{mL}(p<0.001)$. The cellular inflammatory response returned thereafter to near baseline values without any statistical significant difference between the two groups (Fig. 1).

PLGA microspheres were easily identified under light microscopy inside the alveolar macrophages (Fig. 2). On the first day after tracheal instillation, PLGA microspheres were also seen inside some neutrophils. The number and size of PLGA microspheres within the alveolar macrophages decreased gradually with time after tracheal instillation (Figs. 2 and 3) as did the number of macrophages containing microspheres (Fig. 4).
PLGA microspheres were detected up to $58 \mathrm{~d}$ after tracheal instillation (end of study) with a $90 \%$ rate of disappearance of about $36 \mathrm{~d}$ (Fig. 3).

Histologic examination revealed no evidence of inflammation of the lungs after saline instillation. After PLGA instillation, there was a mild peribronchial neutrophilic infiltration on the first day that returned to normal thereafter. No PLGA microspheres were detected in the heart, perihilar lymph glands, liver, and spleen of PLGA-instilled animals. The histology of these organs was normal.

\section{DISCUSSION}

Aspiration of foreign material into the tracheobronchial tree is probably a significant cause for a variety of respiratory disorders. Food or gastric content aspirated into the airways can cause laryngeal, tracheal, and bronchial inflammation resulting in stridor, cough, wheezing, recurrent pneumonia, bronchiectasis, pulmonary fibrosis, and even suffocation and death due to massive aspiration $(3,13,14)$. In neurologically handicapped children, aspiration is common, due either to massive gastroesophageal reflux $(1,2)$ or to aspiration from above due to noncoordination between breathing and swallowing mechanisms (15). Aspiration secondary to swallowing dysfunction may also occur in neurologically normal infants. Sheikh et al. (16) showed that 13 of 112 neurologically normal infants $(11.6 \%)$ who were evaluated for recurrent
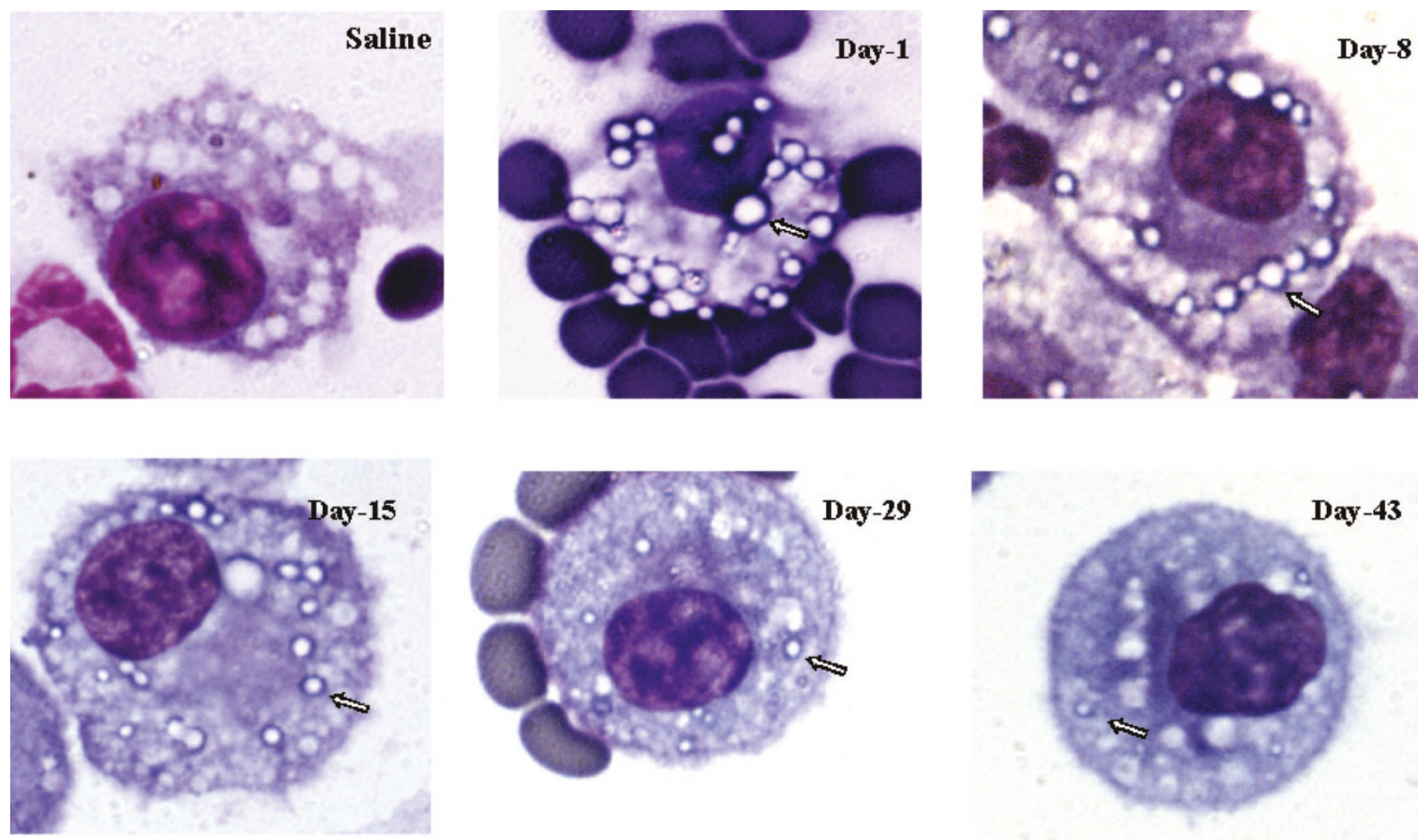

$10 \mu \mathrm{m}$

Figure 2. Microscopic view of PLGA microspheres inside macrophages. PLGA microspheres inside alveolar macrophages (arrows) during days 1, 8, 15, 29, and 43 after a single tracheal instillation of PLGA suspension (original magnification $\times 1000$ ). 


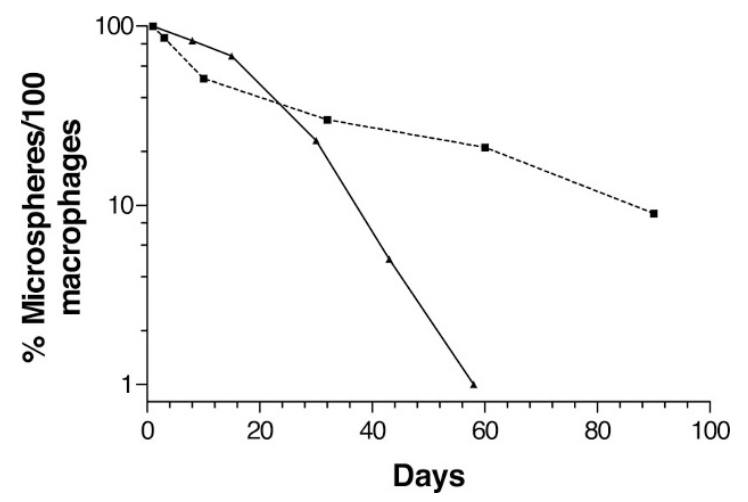

Figure 3. Rate of disappearance of PLGA and polystyrene microspheres. Comparison of the rate of disappearance of PLGA (solid line) and polystyrene (dashed line) microspheres inside alveolar macrophages. PLGA data are from the present study and polystyrene data are from our previous study (10). The amount of microspheres is expressed as the percentage of microspheres relative to the number counted on the first day after tracheal instillation.

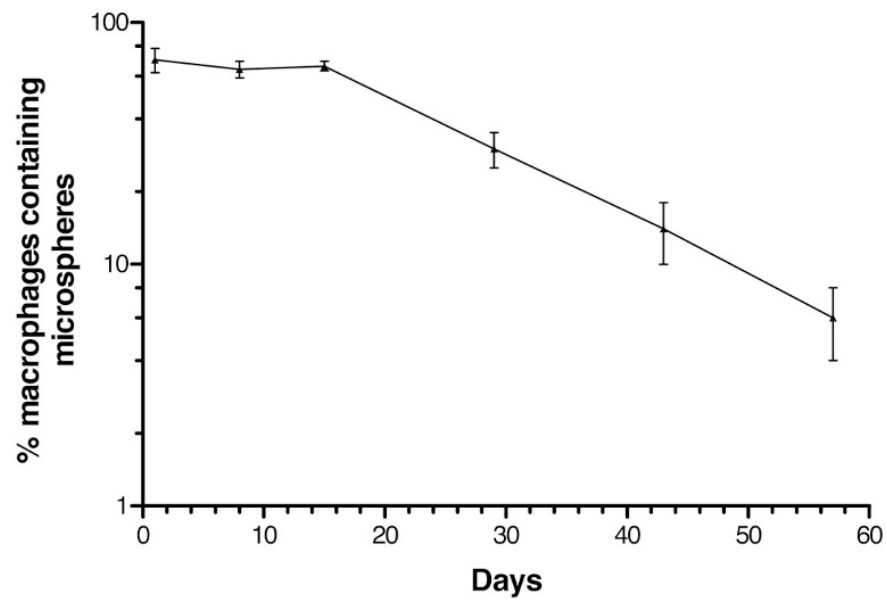

Figure 4. Rate of disappearance of macrophages with PLGA microspheres. Time course of the change in percentage macrophages (mean \pm standard error of the mean) containing PLGA microspheres after a single tracheal instillation of PLGA suspension.

stridor or wheezing had evidence of aspiration on videofluoroscopic swallowing studies.

The definite diagnosis of aspiration is difficult. The commonly used tests such as videofluoroscopic swallowing studies and radionuclide scintigraphy have been found to be quite insensitive (5). Gastroesophageal reflux can be diagnosed by using these techniques and also by 24-h esophageal $\mathrm{pH}$ monitoring (17), echo-Doppler ultrasound (18), and esophageal intraluminal impedance (19). However, these tests are of no use in diagnosing aspiration. The demonstration of lipids in alveolar macrophages had been suggested as a marker for aspiration in early reports by Sundberg et al. (20) and by Williams and Freeman (21). Later, Corwin et al. (6) evaluated bronchoalveolar lavage in adult patients with a variety of parenchymal lung diseases using oil red $\mathrm{O}$ staining to demonstrate intracellular lipids. They showed that aspirators had a significantly higher amount of intracellular lipid vacuoles than nonaspirators. However, they were careful enough to state that "the mere presence of lipid-laden alveolar macrophages in lower respiratory secretions is a nonspecific marker of parenchymal lung disease," and that only "the computation of a
LLAM index may be helpful in excluding aspiration as a cause of parenchymal lung disease." Similar results were found in children in a study by Colombo and Hallberg (22). During some $10 \mathrm{y}$ following the publications of Corwin et al. (6) and Colombo and Hallberg (22), the identification of LLAM by the oil red $\mathrm{O}$ staining method was considered by many to be the gold standard test for the diagnosis of aspiration. However, during the past several years, several investigators have shown that increased numbers of LLAM with indices similar to those found in patients with aspiration can also be found in other diseases without evidence of aspiration (7-9). Elidemir et al. (23) showed in a murine model that aspiration of milk could be diagnosed by immunocytochemical staining of milk proteins ( $\alpha$-lactalbumin and $\beta$-lactoglobulin) in alveolar macrophages. However, as shown in their study, it was possible to detect intracellular milk proteins only during the first $4 \mathrm{~d}$ following aspiration. Recently, Metheny and colleagues demonstrated that aspiration of gastric content could be detected by a specific pepsin immunoassay in tube-fed adults (24) and children (25) receiving mechanical ventilation and in an experimental model in rabbits (26). Although the technique is very sensitive for the diagnosis of aspiration secondary to gastroesophageal reflux, it does not detect aspiration from above due to disturbances such as swallowing mechanism dysfunction.

The need for a simple, specific, and sensitive test to diagnose aspiration led us to search for an exogenous marker that could be mixed with any infant formula, and, if aspirated, would be easily detected in bronchoalveolar lavage for at least a few weeks after a single aspiration episode. In a recent study, we showed that polystyrene microspheres with a diameter of 2.1 $\mu \mathrm{m}$ instilled directly into hamsters' trachea can be easily detected in alveolar macrophages for at least 3 mo after a single instillation (10). Although there were no signs of inflammation or other histologic abnormalities in the lungs or in other internal organs up to $90 \mathrm{~d}$ after the first day of polystyrene microsphere tracheal instillation, we were concerned about the possible long-term effects of polystyrene microspheres in humans, as they are composed of a nondegradable material. In another study, we have recently shown that a suspension of starch granules (corn flour) instilled into hamsters' trachea can be easily identified within alveolar macrophages for at least $14 \mathrm{~d}$ after a single instillation (27). This marker seemed very promising, as starch is a food additive and can be mixed with any infant formula. Unfortunately, we later discovered that starch is spread on surgical gloves and thus pollutes almost all the instruments used in hospitals including bronchoscopes. Until starch-free gloves are widely used, this technique should be abandoned.

In our search for a degradable marker to diagnose aspiration, we chose to investigate the usefulness of PLGA microspheres. PLGA is a polymer composed of $50 \%$ polylactic and $50 \%$ polyglycolic acid. PLGA is already widely used in humans as a biodegradable vehicle to deliver drugs and is approved by the U.S. Food and Drug Administration (11). The biodegradation of PLGA occurs through nonenzymatic hydrolysis into lactic and glycolic acids. The rate of degradation depends on the proportion of lactic acid and glycolic acid within the PLGA molecule and also by the molecular weight of PLGA. Lactic 
acid enters the tricarboxylic acid cycle and is metabolized and eliminated as $\mathrm{CO}_{2}$ and water. Glycolic acid is either excreted unchanged in the urine or enters the Krebs cycle and is also eliminated as $\mathrm{CO}_{2}$ and water (11).

In our present study, we have shown that PLGA (MW 12,000) microspheres with a diameter of 1-2 $\mu \mathrm{m}$ instilled directly into the trachea of hamsters were easily detected inside alveolar macrophages from the first day after tracheal instillation and up to $58 \mathrm{~d}$ thereafter (Fig. 3). The number and size of the microspheres decreased gradually with time (Fig. 2), as did the percentage of macrophages containing PLGA microspheres (Fig. 4). When comparing the rate of disappearance of PLGA microspheres to that of polystyrene microspheres from our previous study (10), it can be seen that $90 \%$ of PLGA microspheres disappeared in $36 \mathrm{~d}$ compared with $88 \mathrm{~d}$ in the polystyrene study (Fig. 3). This difference can be attributed to the degradation of the PLGA microspheres inside the alveolar macrophages.

We have shown that PLGA microspheres were stable in size and number for at least $21 \mathrm{~d}$ in an acidic solution ( $\mathrm{pH}$ 1.0). This makes PLGA an attractive marker also for aspiration secondary to gastroesophageal reflux, as the microspheres will probably not be destroyed by the low $\mathrm{pH}$ of the stomach content.

On the first day after PLGA aspiration, there was an acute inflammatory response, as was demonstrated by an increase in neutrophils in WLL and in lung parenchyma, that disappeared thereafter. A similar inflammatory response was also noted in our earlier studies in hamsters using other markers such as polystyrene microspheres (10) and corn flour (24). This transient inflammation is probably a response to the presence of foreign material instilled into the airways. No histologic abnormalities were detected in other internal organs after PLGA aspiration.

In summary, we have shown that PLGA microspheres (MW 12,000 , with a diameter of 1-2 $\mu \mathrm{m}$ ) are a sensitive and specific marker for the diagnosis of aspiration in hamsters. The usefulness of this test in diagnosing aspiration in humans should be further evaluated in clinical studies.

\section{REFERENCES}

1. Rogers BT, Arvedson J, Msall M, Demerath RR 1993 Hypoxemia during oral feeding with severe cerebral palsy. Dev Med Child Neurol 35:3-10

2. Sondheimer JM, Morris BA 1979 Gastroesophageal reflux among severely retarded children. J Pediatr 94:710-714
3. Bauer ML, Figueroa-Colon R, Georgeson K, Young DW 1993 Chronic pulmonary aspiration in children. South Med J 86:789-795

4. Wilson SL, Thach BT, Brouillette RT, Abu-Osba YK 1981 Coordination of breathing and swallowing in human infants. J Appl Physiol 50:851-858

5. McVeagh P, Howman-Giles R, Kemp A 1987 Pulmonary aspiration studied by radionuclide milk scanning and barium swallow roentgenography. Am J Dis Child 141:917-921

6. Corwin RW, Irwin RS 1985 The lipid-laden alveolar macrophage as a marker of aspiration in parenchymal lung disease. Am Rev Respir Dis 132:576-581

7. Kajetanowicz A, Stinson D, Laybolt KS, Resch L 1999 Lipid-laden macrophages in tracheal aspirate of ventilated neonates receiving intralipid: a pilot study. Pediatr Pulmonol 28:101-108

8. Knauer-Fischer S, Ratjen F 1999 Lipid-laden macrophages in bronchoalveolar lavage fluid as a marker for pulmonary aspiration. Pediatr Pulmonol 27:419-422

9. Vichinsky E, Williams R, Das M, Earles AN, Lewis N, Adler A, McQuitty J 1994 Pulmonary fat embolism: a distinct cause of severe acute chest syndrome in sickle cell anemia. Blood 83:3107-3122

10. Avital A, Shapiro E, Doviner V, Sherman Y, Margel S, Tsuberi M, Springer C 2002 Polystyrene microspheres as a specific marker for the diagnosis of aspiration in hamsters. Am J Respir Cell Mol Biol 27:511-514

11. Rajeev AJ 2000 The manufacturing techniques of various drug loaded biodegradable poly(lactide-co-glycolide) (PLGA) devices. Biomaterials 21:2475-2490

12. Collett JH 2003 Poloxamer. In: Rowe RC, Sheskey PJ, Weller PJ (eds) Handbook of Pharmaceutical Excipients, 4th ed. Pharmaceutical Press, London, pp 447-450

13. Mendelson CL 1946 The aspiration of stomach contents into the lungs during obstetric anesthesia. Am J Obstet Gynecol 52:191-205

14. Moran TJ Experimental aspiration pneumonia. IV. 1955 Inflammatory and reparative changes produced by intratracheal injections of autologous gastric juice and hydrochloric acid. Arch Pathol 60:122-129

15. Arvedson J, Rogers B, Buck G, Smart P, Msall M 1994 Silent aspiration prominent in children with dysphagia. Int J Pediatr Otorhinolaryngol 28:173-181

16. Sheikh S, Allen E, Shell R, Hruschak J, Iram D, Castile R, McCoy K 2001 Chronic aspiration without gastroesophageal reflux as a cause of chronic respiratory symptoms in neurologically normal infants. Chest 120:1190-1195

17. Vandenplas Y, Derde MP, Piepsz A 1992 Evaluation of reflux episodes during simultaneous esophageal $\mathrm{pH}$ monitoring and gastroesophageal reflux scintigraphy in children. J Pediatr Gastroenterol Nutr 14:256-260

18. Hirsch W, Kedar R, Preiss U 1996 Color doppler in the diagnosis of the gastroesophageal reflux in children: comparison with $\mathrm{pH}$ measurements and $\mathrm{B}$-mode ultrasound Pediatr Radiol 26:232-235

19. Skopnik H, Silny J, Heiber O, Schultz J, Rau G, Heimann G 1996 Gastroesophageal reflux in infants: evaluation of a new intraluminal impedance technique. J Pediatr Gastroenterol Nutr 23:591-598

20. Sundberg RH, Kirschner KE, Brown MJ 1959 Evaluation of lipoid pneumonia. Dis Chest 36:594-601

21. Williams HE, Freeman M 1973 Milk inhalation pneumonia: the significance of fat filled macrophages in tracheal secretions. Aust Paediatr J 9:286-288

22. Colombo JL, Hallberg TK 1987 Recurrent aspiration in children: lipid-laden alveolar macrophage quantitation. Pediatr Pulmonol 3:86-89

23. Elidemir O, Fan LL, Colasurdo N 2000 A novel diagnostic method for pulmonary aspiration in a murine model. Immunocytochemical staining of milk proteins in alveolar macrophages. Am J Respir Crit Care Med 161:622-626

24. Metheny NA, Chang YH, Ye JS, Edwards SJ, Defer J, Dahms TE, Stewart BJ, Stone KS, Clouse RE 2002 Pepsin as a marker for aspiration. Am J Crit Care 11:150-154

25. Meert KL, Daphtary KM, Metheny NA 2002 Detection of pepsin and glucose in tracheal secretions as indicators of aspiration in mechanically ventilated children. Pediatr Crit Care Med 3:19-22

26. Metheny NA, Dahms TE, Chang YH, Stewart BJ, Frank PA, Clouse RE 2004 Detection of pepsin in tracheal secretions after forced small-volume aspirations of gastric juice. JPEN J Parenter Enteral Nutr 28:79-84

27. Springer C, Avital A 2003 Simple and specific test for the diagnosis of aspiration into the airway using a corn flour-milk mixture in a hamster model. Pediatr Pulmonol 35:400-4004 